\title{
Uncertain Portfolio Selection with Background Risk and Liquidity Constraint
}

\author{
Jia Zhai and Manying Bai \\ School of Economics and Management, Beihang University, Beijing 100191, China \\ Correspondence should be addressed to Jia Zhai; zhaijia891115@sina.com
}

Received 8 October 2016; Revised 29 November 2016; Accepted 14 December 2016; Published 22 January 2017

Academic Editor: Thomas Hanne

Copyright ( 2017 Jia Zhai and Manying Bai. This is an open access article distributed under the Creative Commons Attribution License, which permits unrestricted use, distribution, and reproduction in any medium, provided the original work is properly cited.

\begin{abstract}
This paper discusses an uncertain portfolio selection problem with consideration of background risk and asset liquidity. In addition, the transaction costs are also considered. The security returns, background asset return, and asset liquidity are estimated by experienced experts instead of historical data. Regarding them as uncertain variables, a mean-risk model with background risk, liquidity, and transaction costs is proposed for portfolio selection and the crisp forms of the model are provided when security returns obey different uncertainty distributions. Moreover, for better understanding of the impact of background risk and liquidity on portfolio selection, some important theorems are proved. Finally, numerical experiments are presented to illustrate the modeling idea.
\end{abstract}

\section{Introduction}

Portfolio selection discusses the problem of how to allocate one's capital in different risky securities to maximize the return of portfolio for a given risk. Markowitz [1] first proposed the mean-variance model in 1952, which laid the foundation of modern portfolio theory and has been the most impact-making development in mathematical finance management. However, measuring the risk by the variance of return of a portfolio has some limitations and there is computational difficulty to construct large-scale portfolio. In view of this, scholars studied other methods to measure investment risk of the portfolio and built a lot of portfolio optimization models, such as mean-semivariance model [2, 3], expected absolute deviation model [4], Value-at-Risk model [5], Conditional Value-at-Risk models [6], meansemivariance-CVaR model [7], and mean-risk curve model [8]. In this paper, we will use risk curve defined in uncertain environment [9] as the risk measurement.

In real life, all the relevant information for an investment decision cannot be confined in terms of return and risk. Portfolio selection models that take into account more criteria other than the return and risk have become well liked. Transaction cost is one of the main concerns for portfolio managers. Arnott and Wagner [10] found that ignoring transaction costs would lead to an inefficient portfolio. In some situations, investors may consider other factors such as liquidity. Liquidity has been measured as the degree of chance involved in the conversation of an investment into cash without any significant loss in value. Parra et al. [11] proposed the model that consists of three criteria: return, risk, and liquidity. Fang et al. [12] presented a portfolio rebalancing model with transaction costs based on fuzzy decision theory considering three criteria: return, risk, and liquidity. Thus, transaction costs and liquidity are considered in the portfolio selection model we will discuss.

These researches assume that all that investors face only portfolio risk when making portfolio selection decisions. Yet, in reality, investors also face other sources of risk like labor income, investments in real estate, and unexpected expenses related to health issues that cannot be traded in the financial market; these sources of risk are usually referred to as background risk $[13,14]$. To help investors select portfolio in face of background risk, Baptista offered conditions for investors to optimally delegate their wealth management to portfolio managers in face of background risk [15]. We refer to the assets that are exposed to background risk as background assets. Investors concern not only the risk of 
financial assets within portfolio but also the background risk. Many studies have showed that the presence of background risk can affect investments [16]. For example, Heaton and Lucas found that labor and entrepreneurial incomes affected portfolio selection [17]. Rosen and Wu showed that investors with bad health were more willing to put most of their capital in the low risk asset rather than risky assets [18]. Some studies reveal that investors who consider background risk will become more risk averse and prefer to choose safer assets [19]. Therefore, a portfolio selection model where background risk is considered will be discussed in this paper.

In portfolio theory, the security returns are generally considered as random variables. However, there may be lack of enough historical data in some emerging markets. Particularly, for background asset returns, there is much subjective impression rather than randomness; then if people still use probability theory to solve this problem, counterintuitive results may occur $[20,21]$. In the situation, scholars found that the security returns data and background asset returns can be estimated by experienced experts and fuzzy set theory [22] can be used to deal with this problem. Then, fuzzy theory is also applied to portfolio selection [23, 24]. Huang established the mean-variance model [25] and mean-semivariance [26] model for portfolio selection in fuzzy environment. Qin et al. presented fuzzy cross-entropy method for portfolio selection [27]. In 2010, Li et al. formulated mean-variance-skewness models [28] which considered the skewness to measure the asymmetry of fuzzy portfolio return. Furthermore, Fuzzy mean-variance-skewness portfolio selection model by interval analysis which considers some criteria like transaction costs, liquidity, and so forth was proposed in 2011 [29]. Although fuzzy portfolio optimization provided alternatives to estimate security returns with lack of data, fuzzy theory suffers from criticism since a paradox will appear. In order to better describe the subjective imprecise quantity, in 2007, Liu developed uncertainty theory [30]. Based on this framework, Huang introduced uncertainty theory to portfolio selection and produced an uncertain portfolio theory [31]. Later, Huang found that risk curve is a good way to measure risk in uncertain environment and built a meanrisk model. In 2012, Huang established a risk index model [32] and a mean-variance model [33] for portfolio selection and the security returns were given by experts' evaluations. For optimal project selection and schedule, Huang et al. presented mean-semivariance model [34] and mean-chance model [35] based on uncertain measure. Moreover, the meanvariance model for portfolio optimization problem in the simultaneous presence of random and uncertain returns was proposed by Qin [36]. Numerous studies have been done about uncertain portfolio selection but a few papers consider background risk and regard background asset returns as uncertain variables. Until 2016, Huang and Di researched the model with background risk for portfolio selection [37].

In this paper, risk curve will be employed to measure investment risk since it provides information about all the likely losses, and security returns, background asset returns, and asset liquidity will be discussed in uncertain environment. Therefore, a new mean-risk model with background risk for uncertain portfolio selection will be proposed and transaction costs and liquidity are considered. We analyze some important properties in order to illustrate the effect of background risk and liquidity on portfolio selection. Our model is compared with the model in Huang and Di's paper [37] with transaction costs and asset liquidity.

The remainder of the paper is organized as follows. Section 2 in detail describes the model we proposed for portfolio selection based on uncertainty theory and gives some important theorems. In Section 3, we employ the numerical examples to illustrate the validity and significance of the model. Section 4 lists the conclusions. In Appendix, we review the necessary knowledge about uncertainty theory.

\section{The Portfolio Selection Models}

2.1. Basic Conceptions. Investors' uncertain return includes both uncertain portfolio return from financial assets and uncertain return from background assets. When these asset returns are given by experts' estimations, it is better to use uncertain variables to describe them. Since all the background assets have same features that are different from financial assets, that is, nontradable and unhedgeable, the popular way is to use one parameter $r_{b}$ to show the returns from all the background assets in real life. In addition, we assume that the background asset return rate has zero expected value. The assumption that nontradable background asset has zero expected return rate, which is in consistent with assumptions of most researches studying portfolio with background risk $[15,38]$. The model we will propose is also applicable in the situation where the expected value of the background asset return rate differs from zero.

Firstly, the definitions of risk curve and confidence curve will be introduced as follows.

Definition 1 (see [9]). Let $\xi$ be an uncertain return rate of a security and $r_{f}$ the risk-free interest rate. Then the curve

$$
R(r)=M\left\{r_{f}-\xi \geq r\right\}, \quad \forall r \geq 0,
$$

is called the risk curve of the security.

Since all investors know that they may lose as well as gain in investment, they will have a maximum tolerance towards occurrence chance of each likely loss level; we call it confidence curve $\alpha(r)$. A portfolio is safe if its risk curve is below the confidence curve and a portfolio is risky if any part of its risk curve is above the confidence curve.

2.2. Assumptions and Notations. Let us consider a financial market with $n$ risky assets. An investor allocates his total capital among the risky assets. For the $i$ th risky asset $(i=$ $1,2, \ldots, n)$ and background assets, the following notations will be used:

$x_{i}$ : the proportion of the total capital invested in security $i$

$p_{i}$ : the closing price of the $i$ th security at present

$p_{i}^{\prime}$ : the estimated closing price of the $i$ th security in the next month 
$d_{i}$ : the estimated dividend of the $i$ th security during the month

$\xi_{i}:\left(p_{i}^{\prime}+d_{i}-p_{i}\right) / p_{i}=$ the return rate of the $i$ th security $r_{b}$ : the returns from all background assets

$c_{i}$ : the transaction cost

$k_{i}$ : the constant cost per change on a proportion, $k_{i} \geq$ 0

$\eta_{i}$ : the turnover rate of the $i$ th security

$r_{f}$ : risk-free interest rate

2.3. The Uncertain Models. Let us consider the transaction cost $c_{i}$ to be a V-shape function of the difference between a given portfolio $x^{0}=\left(x_{1}^{0}, x_{2}^{0}, \ldots, x_{n}^{0}\right)$ and a new portfolio $x=\left(x_{1}, x_{2}, \ldots, x_{n}\right)$ and it is incorporated explicitly into the portfolio return. Thus the transaction cost of the ith risky asset can be expressed as

$$
c_{i}=k_{i}\left|x_{i}-x_{i}^{0}\right|, \quad i=1,2, \ldots, n .
$$

Hence the total transaction cost is

$$
\sum_{i=1}^{n} c_{i}=\sum_{i=1}^{n} k_{i}\left|x_{i}-x_{i}^{0}\right|
$$

Then the expected return of the portfolio $x=\left(x_{1}, x_{2}, \ldots, x_{n}\right)$ with transaction cost is given by

$$
E\left(\xi_{1} x_{1}+\xi_{2} x_{2}+\cdots+\xi_{n} x_{n}+r_{b}\right)-\sum_{i=1}^{n} k_{i}\left|x_{i}-x_{i}^{0}\right| .
$$

For a new investor it can be assumed that $x_{i}^{0}=0, i=$ $1,2, \ldots, n$. We consider that the investor is a new investor and he has no security on hand in the models below.

The investor's total return which includes the portfolio return and background assets return can be expressed as $r_{p}=$ $\sum_{i=1}^{n} \xi_{i} x_{i}+r_{b}-\sum_{i=1}^{n} k_{i} x_{i}$. Let us define the risk curve of the portfolio as follows:

$$
\begin{array}{r}
R\left(x_{1}, x_{2}, \ldots, x_{n}, r_{b}, k_{1}, \ldots, k_{n} ; r\right) \\
=M\left\{r_{f}-\left(\xi_{1} x_{1}+\cdots+\xi_{n} x_{n}+r_{b}-\sum_{i=1}^{n} k_{i} x_{i}\right) \geq r\right\}, \\
\forall r \geq 0,
\end{array}
$$

where the expected value of $r_{b}$ is 0 and the variance value is $\rho$. The background assets are independent of other $n$ risky assets.

Liquidity is the degree of the chance of converting an investment into cash without any significant loss in value. In general, the securities liquidity may be reflected by turnover rate. Turnover rate is the number of shares traded divided by the number of shares outstanding in that stock and think of it as an intuitive metric of the liquidity of the stock. Investors usually prefer greater liquidity. It is known that turnover rates of the securities in the future cannot be predicted accurately. Therefore, we regard turnover rates as uncertain variables.
Keeping in mind what we have discussed above, for a new investor, the uncertain portfolio selection model is constructed as follows:

$$
\begin{array}{ll}
\max & E\left[\xi_{1} x_{1}+\xi_{2} x_{2}+\cdots+\xi_{n} x_{n}+r_{b}\right]-\sum_{i=1}^{n} k_{i} x_{i} \\
\text { s.t. } & R\left(x_{1}, x_{2}, \ldots, x_{n}, r_{b}, k_{1}, \ldots, k_{n} ; r\right) \leq \alpha(r), \quad \forall r \geq 0, \\
& E\left[\sum_{i=1}^{n} \eta_{i} x_{i}\right] \geq l, \\
& \sum_{i=1}^{n} x_{i}=1, \\
& x_{i} \geq 0, i=1,2, \ldots, n .
\end{array}
$$

The first constraint ensures the portfolio is safe, and the second one assures that liquidity is no less than some given level $l$, where $\alpha(r)$ and $l$ will be allocated by the investor.

The variation of model (6) is the following:

$$
\begin{array}{ll}
\max & E\left[\xi_{1} x_{1}+\xi_{2} x_{2}+\cdots+\xi_{n} x_{n}+r_{b}\right]-\sum_{i=1}^{n} k_{i} x_{i} \\
\text { s.t. } & R\left(x_{1}, x_{2}, \ldots, x_{n}, r_{b}, k_{1}, \ldots, k_{n} ; r\right) \leq \alpha(r), \quad \forall r \geq 0, \\
& M\left\{\sum_{i=1}^{n} \eta_{i} x_{i} \geq l\right\} \geq \beta, \\
& \sum_{i=1}^{n} x_{i}=1, \\
& x_{i} \geq 0, i=1,2, \ldots, n,
\end{array}
$$

where $M$ denotes the uncertain measure and the second constraint tells that the chance of the liquidity exceeding a threshold level $l$ should not be less than a preset tolerable level $\beta$.

\subsection{Deterministic Forms of the Uncertain Model}

Theorem 2. Let $\Phi_{i}$ denote the continuous and strictly increasing uncertainty distribution of the ith security return rate $\xi_{i}$ whose inverse function $\Phi_{i}^{-1}(\alpha(r))$ exists and is unique for each $\alpha(r) \in(0,1), i=1,2, \ldots, n$, respectively. Suppose the uncertain return rate of background asset $r_{b}$ has continuous and strictly increasing uncertainty distribution function $\Theta$. Then the meanrisk model with background risk (6) can be transformed into the following linear model:

$$
\begin{array}{ll}
\max & x_{1} E\left[\xi_{1}\right]+x_{2} E\left[\xi_{2}\right]+\cdots+x_{n} E\left[\xi_{n}\right]-\sum_{i=1}^{n} k_{i} x_{i} \\
\text { s.t. } & x_{1} \Phi_{1}^{-1}(\alpha(r))+x_{2} \Phi_{2}^{-1}(\alpha(r))+\cdots \\
& +x_{n} \Phi_{n}^{-1}(\alpha(r))+\Theta^{-1}(\alpha(r))-\sum_{i=1}^{n} k_{i} x_{i} \\
& \geq r_{f}-r, \quad \forall r \geq 0,
\end{array}
$$




$$
\begin{aligned}
& E\left[\sum_{i=1}^{n} \eta_{i} x_{i}\right] \geq l, \\
& \sum_{i=1}^{n} x_{i}=1, \\
& x_{i} \geq 0, i=1,2, \ldots, n .
\end{aligned}
$$

Proof. It follows from Theorem A.9 that the objective function of model (6) can be transformed into the objective function of model (8).

Since

$$
\begin{aligned}
& R\left(x_{1}, x_{2}, \ldots, x_{n}, r_{b}, k_{1}, \ldots, k_{n} ; r\right)=M\left\{r_{f}\right. \\
& \left.\quad-\left(\xi_{1} x_{1}+\xi_{2} x_{2}+\cdots+\xi_{n} x_{n}+r_{b}-\sum_{i=1}^{n} k_{i} x_{i}\right) \geq r\right\} \\
& \quad=M\left\{\xi_{1} x_{1}+\xi_{2} x_{2}+\cdots+\xi_{n} x_{n}+r_{b} \leq r_{f}-r\right. \\
& \left.\quad+\sum_{i=1}^{n} k_{i} x_{i}\right\}
\end{aligned}
$$

then according to the monotonicity property of uncertain variable, it can be found that

$$
\begin{gathered}
x_{1} \Phi_{1}^{-1}(\alpha(r))+x_{2} \Phi_{2}^{-1}(\alpha(r))+\cdots+x_{n} \Phi_{n}^{-1}(\alpha(r)) \\
+\Theta^{-1}(\alpha(r))-\sum_{i=1}^{n} k_{i} x_{i} \geq r_{f}-r .
\end{gathered}
$$

The theorem is completed.

Next, model (8) will be converted into the following forms when the distribution of security return rates is determined.

Theorem 3. Suppose the return rates of the ith securities are all normal uncertain variables $\xi_{i} \sim N\left(\mu_{i}, \sigma_{i}\right), i=1,2, \ldots, n$. The return rate of background asset is $r_{b}$ and $r_{b} \sim N(0, \rho)$. Then model (8) can be transformed into the following form:

$$
\begin{array}{cl}
\max \quad & x_{1} \mu_{1}+x_{2} \mu_{2}+\cdots+x_{n} \mu_{n}-\sum_{i=1}^{n} k_{i} x_{i} \\
\text { s.t. } \quad & \sum_{i=1}^{n}\left(\mu_{i}-\frac{\sqrt{3} \sigma_{i}}{\pi} \ln \frac{1-\alpha(r)}{\alpha(r)}\right) \cdot x_{i} \\
& -\frac{\sqrt{3} \rho}{\pi} \ln \frac{1-\alpha(r)}{\alpha(r)}-\sum_{i=1}^{n} k_{i} x_{i} \geq r_{f}-r,
\end{array}
$$

$\forall r \geq 0$,

$$
\begin{aligned}
& E\left[\sum_{i=1}^{n} \eta_{i} x_{i}\right] \geq l, \\
& \sum_{i=1}^{n} x_{i}=1, \\
& x_{i} \geq 0, \quad i=1,2, \ldots, n .
\end{aligned}
$$

Theorem 4. Suppose the return rates of the ith securities are all zigzag uncertain variables $\xi_{i} \sim z\left(a_{i}, b_{i}, c_{i}\right), i=1,2, \ldots, n$. The return rate of background asset is $r_{b}$ and $r_{b} \sim N(0, \rho)$. Then model (8) can be transformed into the following form.

When $\alpha(r) \in(0,1 / 2]$,

$$
\begin{array}{ll}
\max & \sum_{i=1}^{n} \frac{a_{i}+2 b_{i}+c_{i}}{4} x_{i}-\sum_{i=1}^{n} k_{i} x_{i} \\
\text { s.t. } & \sum_{i=1}^{n} 2 \alpha(r)\left(b_{i}-a_{i}\right) x_{i}+\sum_{i=1}^{n} a_{i} x_{i} \\
& -\frac{\sqrt{3} \rho}{\pi} \ln \frac{1-\alpha(r)}{\alpha(r)}-\sum_{i=1}^{n} k_{i} x_{i} \geq r_{f}-r, \\
& E\left[\sum_{i=1}^{n} \eta_{i} x_{i}\right] \geq l, \\
& \sum_{i=1}^{n} x_{i}=1, \\
& x_{i} \geq 0, i=1,2, \ldots, n .
\end{array}
$$

\subsection{Discussion of the Uncertain Models}

Theorem 5. For any given $r$ and confidence curve $\alpha(r)(0<$ $\alpha(r)<0.5)$, the expected return of the optimal portfolio with background risk is smaller than that without background risk.

Proof. When no background risk is considered, model (8) becomes of the following form:

$$
\begin{array}{ll}
\max & x_{1} E\left[\xi_{1}\right]+x_{2} E\left[\xi_{2}\right]+\cdots+x_{n} E\left[\xi_{n}\right]-\sum_{i=1}^{n} k_{i} x_{i} \\
\text { s.t. } & x_{1} \Phi_{1}^{-1}(\alpha(r))+x_{2} \Phi_{2}^{-1}(\alpha(r))+\cdots \\
& +x_{n} \Phi_{n}^{-1}(\alpha(r))-\sum_{i=1}^{n} k_{i} x_{i} \geq r_{f}-r, \quad \forall r \geq 0, \\
& E\left[\sum_{i=1}^{n} \eta_{i} x_{i}\right] \geq l, \\
& \sum_{i=1}^{n} x_{i}=1, \\
& x_{i} \geq 0, i=1,2, \ldots, n .
\end{array}
$$


Since $E\left(r_{b}\right)=0$, the objective function of model (13) is same as that of model (8).

Suppose $X=\left(x_{1}, x_{2}, \ldots, x_{n}\right)$ is the optimal solution of model (8), then for any given $r \geq 0$, we have

$$
\begin{aligned}
f_{1}(X, \alpha(r))= & x_{1} \Phi_{1}^{-1}(\alpha(r))+x_{2} \Phi_{2}^{-1}(\alpha(r))+\cdots \\
& +x_{n} \Phi_{n}^{-1}(\alpha(r))+\Theta^{-1}(\alpha(r))-\sum_{i=1}^{n} k_{i} x_{i} \\
= & r_{f}-r \\
f_{2}(X)= & E\left[\sum_{i=1}^{n} \eta_{i} x_{i}\right]=l .
\end{aligned}
$$

Let $g_{1}(X, \alpha)$ and $g_{2}(X)$ be the first constraint and second constraint of model (13), respectively. In real life, many investors only care about $0<\alpha(r)<0.5$, because investors' tolerance level should not be larger than 0.5 , because " $\alpha(r)>$ 0.5 " means the investors know that the chance of the occurrence of risk is very large; then they cannot tolerate. So $\alpha(r)<0.5$ and $\Theta^{-1}(\alpha(r))<0$. Then

$$
\begin{aligned}
g_{1}(X, \alpha) & =f_{1}(X, \alpha)-\Theta^{-1}(\alpha(r))>r_{f}-r, \\
g_{2}(X) & =f_{2}(X)=l .
\end{aligned}
$$

That is to say, $X$ is a feasible solution of model (13).

In a similar way, let $X^{\prime}$ be the optimal solution of model (13); it is easy to prove that $X^{\prime}$ is not the feasible solution of model (8). Thus, we can know that $X \neq X^{\prime}$. Since the objective functions of model (8) and model (13) are same, thus for a given $r$ value the expected return of the optimal portfolio with background risk is smaller than that without background risk.

Theorem 6. For any given $r$, the expected value of the optimal portfolio with liquidity is not larger than that without liquidity.

Proof. When no liquidity is considered, model (8) becomes of the following form:

$$
\begin{array}{ll}
\max & x_{1} E\left[\xi_{1}\right]+x_{2} E\left[\xi_{2}\right]+\cdots+x_{n} E\left[\xi_{n}\right]-\sum_{i=1}^{n} k_{i} x_{i} \\
\text { s.t. } \quad & x_{1} \Phi_{1}^{-1}(\alpha(r))+x_{2} \Phi_{2}^{-1}(\alpha(r))+\cdots \\
& \quad+x_{n} \Phi_{n}^{-1}(\alpha(r))+\Theta^{-1}(\alpha(r))-\sum_{i=1}^{n} k_{i} x_{i} \\
& \geq r_{f}-r, \quad \forall r \geq 0, \\
& \sum_{i=1}^{n} x_{i}=1, \\
& x_{i} \geq 0, i=1,2, \ldots, n .
\end{array}
$$

The objective function of model (8) is same as that of model (16). Let $E_{1}$ and $E_{2}$ be the optimal expected value of model (8) and model (16), respectively; the corresponding optimal solution is $X=\left(x_{1}, x_{2}, \ldots, x_{n}\right)$ and $X^{\prime}=$ $\left(x_{1}^{\prime}, x_{2}^{\prime}, \ldots, x_{n}^{\prime}\right)$. We will prove $E_{1} \leq E_{2}$ by using proof by contradiction.

Suppose $E_{1}>E_{2}$; since $X$ is the optimal solution of model (8), then

$$
\begin{aligned}
& f_{1}(X, \alpha(r))= x_{1} \Phi_{1}^{-1}(\alpha(r))+x_{2} \Phi_{2}^{-1}(\alpha(r))+\cdots \\
&+x_{n} \Phi_{n}^{-1}(\alpha(r))+\Theta^{-1}(\alpha(r))-\sum_{i=1}^{n} k_{i} x_{i} \\
&= r_{f}-r \\
& f_{2}(X)=E\left[\sum_{i=1}^{n} \eta_{i} x_{i}\right]=l .
\end{aligned}
$$

Obviously, $X$ is the optimal solution of model (16) and the optimal expected value is $E_{1}$. But $E_{1}>E_{2}$, so there is a contradiction with the fact that $E_{2}$ is the optimal expected value of model (16). Thus, we should have $E_{1} \leq E_{2}$. Theorem is proved.

If liquidity and transaction cost are considered in the model of Huang and Di's paper [37], then the model in Huang and Di's paper is converted into the following form:

$$
\begin{array}{ll}
\max \quad & E\left[\xi_{1} x_{1}+\xi_{2} x_{2}+\cdots+\xi_{n} x_{n}+r_{b}\right]-\sum_{i=1}^{n} k_{i} x_{i} \\
\text { s.t. } \quad & x_{1} \Phi_{1}^{-1}(\alpha)+x_{2} \Phi_{2}^{-1}(\alpha)+\cdots+x_{n} \Phi_{n}^{-1}(\alpha) \\
& +\Theta^{-1}(\alpha)-\sum_{i=1}^{n} k_{i} x_{i} \geq H \\
& E\left[\sum_{i=1}^{n} \eta_{i} x_{i}\right] \geq l \\
& \sum_{i=1}^{n} x_{i}=1, \\
& x_{i} \geq 0, i=1,2, \ldots, n
\end{array}
$$

where $\alpha$ and $H$ are given values.

Theorem 7. The optimal solution of model (8) is also the optimal solution of model (18), but not vice versa.

Proof. If $r$ is a deterministic value, model (8) can degenerate model (18). It is obvious that the optimal solution of model (18) is only one solution of model (8) for a given $r$ but is not always the optimal solution of model (8).

Theorem 8. For any given $r$ and confidence curve $\alpha(r)(0<$ $\alpha(r)<0.5)$, the expected value of the optimal portfolio with background risk and liquidity constraint is smaller than that without background risk and liquidity constraint. 
Proof. When no background risk and liquidity is considered, model (8) becomes of the following form:

$$
\begin{array}{ll}
\max & x_{1} E\left[\xi_{1}\right]+x_{2} E\left[\xi_{2}\right]+\cdots+x_{n} E\left[\xi_{n}\right]-\sum_{i=1}^{n} k_{i} x_{i} \\
\text { s.t. } & x_{1} \Phi_{1}^{-1}(\alpha(r))+x_{2} \Phi_{2}^{-1}(\alpha(r))+\cdots \\
& +x_{n} \Phi_{n}^{-1}(\alpha(r))-\sum_{i=1}^{n} k_{i} x_{i} \geq r_{f}-r, \quad \forall r \geq 0, \\
& \sum_{i=1}^{n} x_{i}=1 \\
& x_{i} \geq 0, i=1,2, \ldots, n .
\end{array}
$$

Since $E\left(r_{b}\right)=0$, the objective function of model (19) is same as that of model (8).

(i) Suppose $X=\left(x_{1}, x_{2}, \ldots, x_{n}\right)$ is the optimal solution of model (8); then for any given $r \geq 0$, we have

$$
\begin{aligned}
& f_{1}(X, \alpha(r))= x_{1} \Phi_{1}^{-1}(\alpha(r))+x_{2} \Phi_{2}^{-1}(\alpha(r))+\cdots \\
&+x_{n} \Phi_{n}^{-1}(\alpha(r))+\Theta^{-1}(\alpha(r)) \\
&-\sum_{i=1}^{n} k_{i} x_{i}=r_{f}-r \\
& f_{2}(X)=E\left[\sum_{i=1}^{n} \eta_{i} x_{i}\right]=l .
\end{aligned}
$$

Let $g_{1}(X, \alpha)$ be the first constraint of model (19), because the expected value of $r_{b}$ is zero. In real life, investors' tolerance level should not be larger than 0.5 . Then $\alpha(r)<0.5$ and $\Theta^{-1}(\alpha(r))<0$; we obtain

$$
g_{1}(X, \alpha)=f_{1}(X, \alpha)-\Theta^{-1}(\alpha(r))>r_{f}-r .
$$

That is to say, $X$ is a feasible solution of model (19).

(ii) Let $X^{\prime}$ be the optimal solution of model (19); then for any given $r \geq 0$, we have

$$
\begin{aligned}
g_{1}\left(X^{\prime}, \alpha\right) & =r_{f}-r \\
f_{1}\left(X^{\prime}, \alpha\right) & =g\left(X^{\prime}, \alpha\right)+\Theta^{-1}(\alpha(r)) \\
& =r_{f}-r+\Theta^{-1}(\alpha(r))<r_{f}-r .
\end{aligned}
$$

So, $X^{\prime}$ is not the feasible solution of model (8).

Thus, from (i) and (ii) we can know that $X \neq X^{\prime}$. Since the objective functions of model (8) and model (19) are the same, then it is easy to get that the expected return of uncertain portfolio selection with background risk and liquidity is smaller than that without background risk and liquidity. Theorem 8 is proved.
TABLE 1: Normal uncertain return rates of 10 securities.

\begin{tabular}{lc}
\hline Security $i$ & Uncertain return rate $\xi_{i}$ \\
\hline 1 & $N(0.027,0.14)$ \\
2 & $N(0.033,0.19)$ \\
3 & $N(0.032,0.16)$ \\
4 & $N(0.039,0.20)$ \\
5 & $N(0.031,0.15)$ \\
6 & $N(0.028,0.15)$ \\
7 & $N(0.030,0.08)$ \\
8 & $N(0.032,0.18)$ \\
9 & $N(0.025,0.10)$ \\
10 & $N(0.026,0.06)$ \\
\hline
\end{tabular}

\section{Numerical Experiment}

In this section, numerical examples are presented to illustrate the proposed uncertain model with background risk and liquidity constraint for portfolio selection and to show the effect of the background risk and liquidity on the portfolio selection decision. The following results are programmed in Matlab 2009a.

Suppose that the turnover rate of the ith security is uncertain variable and the uncertainty distribution function is in the following form:

$$
\Phi(x)= \begin{cases}0, & \text { if } x<s \\ \frac{x-s}{2(t-s)}, & \text { if } s \leq x \leq t \\ \frac{1}{2}, & \text { if } t \leq x \leq u \\ \frac{1}{2}+\frac{x-u}{2(v-u)}, & \text { if } u \leq x \leq v \\ 1, & \text { if } x>v,\end{cases}
$$

where $s, t, u$, and $v$ are real numbers with $s<t<u<v$, denoted by $S(s, t, u, v)$.

Then the turnover rate of the portfolio $x=\left(x_{1}, x_{2}, \ldots, x_{n}\right)$ is $\sum_{i=1}^{n} \eta_{i} x_{i}=\sum_{i=1}^{n}\left(s_{i}, t_{i}, u_{i}, v_{i}\right) x_{i}$. The liquidity is measured by $L(x)=E\left(\sum_{i=1}^{n} \eta_{i} x_{i}\right)=\sum_{i=1}^{n}\left(s_{i}+t_{i}+u_{i}+v_{i}\right) x_{i} / 4$.

Example 1. We select ten stocks. The data we select is from the paper [9]. The estimations of the security return rates and turnover rates are given in Tables 1 and 2, respectively.

Suppose the monthly risk-free interest rate is $0.01, l=$ $0.025, k_{i}=0.001$, and $x_{i}^{0}=0$ for $i=1,2, \ldots, 10$. The experts believe that the background asset return rate has uncertainty distribution $r_{b} \sim N(0,0.01)$, and the investor gives the confidence curve as follows:

$$
\alpha(r)= \begin{cases}-2.75 r+0.43, & 0 \leq r \leq 0.12 \\ -0.5 r+0.16, & 0.12 \leq r \leq 0.3 \\ 0.01, & r \geq 0.3\end{cases}
$$


TABLE 2: Turnover rates of 10 securities.

\begin{tabular}{lc}
\hline Security $i$ & Turnover rate $\eta_{i}$ \\
\hline 1 & $(0.011,0.019,0.009,0.018)$ \\
2 & $(0.009,0.024,0.008,0.025)$ \\
3 & $(0.007,0.016,0.0064,0.018)$ \\
4 & $(0.013,0.032,0.011,0.030)$ \\
5 & $(0.008,0.015,0.0076,0.017)$ \\
6 & $(0.011,0.026,0.009,0.028)$ \\
7 & $(0.012,0.031,0.011,0.030)$ \\
8 & $(0.006,0.031,0.005,0.026)$ \\
9 & $(0.011,0.05,0.0095,0.047)$ \\
10 & $(0.008,0.025,0.006,0.026)$ \\
\hline
\end{tabular}

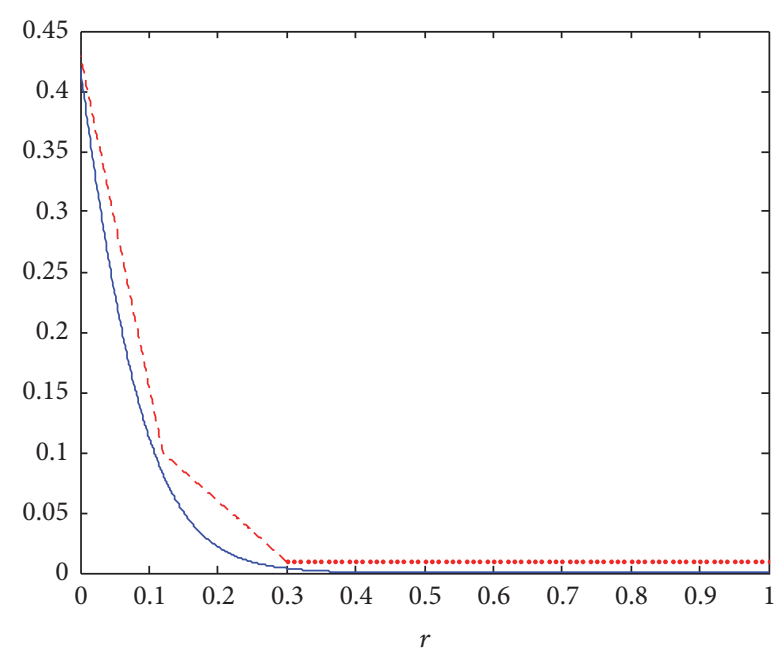

FIGURE 1: Confidence curve and risk curve when background risk and liquidity are considered.

In sight of models (11), (13), and (16) in Section 2, we discuss the optimal portfolio when security return rates are normal uncertain variables and calculate allocation proportions of ten securities to maximize corresponding expected return rate. Set $r \in[0,0.3]$ in the model and let $r=$ $0,0.03,0.06, \ldots, 0.3$, respectively. The optimal portfolios in three different situations can be shown in detail in Table 3.

The expected optimal portfolios with background risk and liquidity, with background risk but without liquidity, and with liquidity but without background risk are $2.78 \%, 3.10 \%$, and $2.86 \%$, respectively. In addition, we have checked that the risk curves of optimal portfolio of three different situations are below the confidence curve; that is, corresponding portfolio is in safe area. Actually, higher expected return rates than the optimal expected value can be found in three situations, but part of their risk curves is above the confidence curve; that is, corresponding portfolio is in risky area.

In Figures 1, 2, 3, and 4, (1) the blue broken line represents confidence curve; (2) the red curve represents risk curve. It can be seen that risk curve is below the confidence curve. Figure 1 shows the relationship between risk curve and confidence curve of the model where background risk and liquidity are considered.

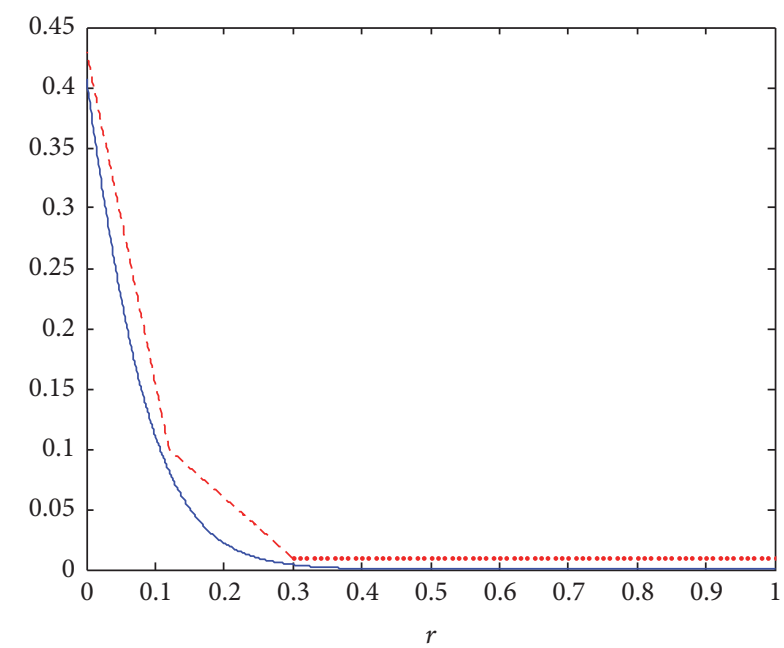

FIGURE 2: Confidence curve and risk curve of the model with background risk but without liquidity.

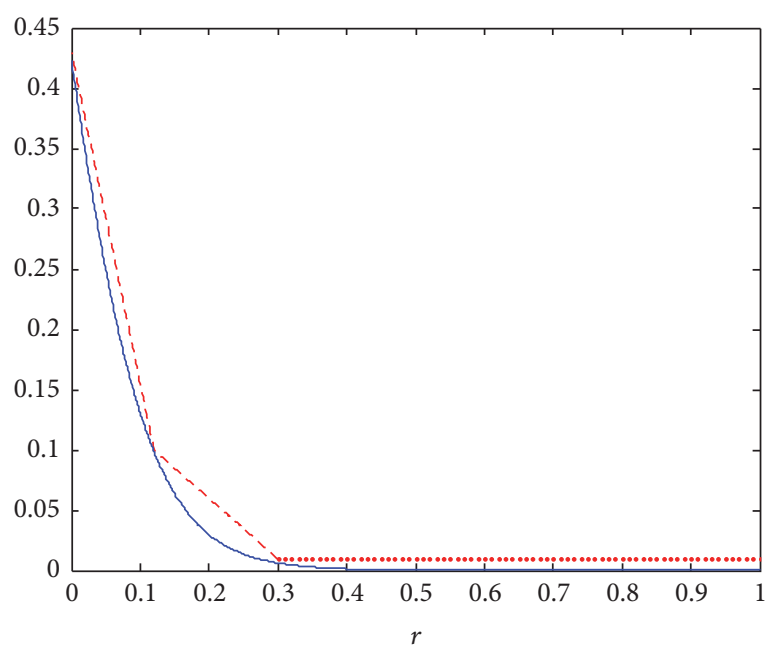

FIGURE 3: Confidence curve and risk curve of the model with liquidity but without background risk.

Similarly, Figure 2 shows the relationship between risk curve and confidence curve of the model where background risk is considered but no liquidity is considered; Figure 3 shows the relationship between risk curve and confidence curve of the model where liquidity is considered but no background risk is considered.

Example 2. Using the data in Tables 1 and 2, we can compute the optimal solution of model (18) when security return rates are normal uncertain variables. In this model, let $\alpha=0.15$, $H=-0.08, \rho=0.01, l=0.025, k_{i}=0.001$, and $x_{i}^{0}=0$ for $i=$ $1,2, \ldots, 10$. Corresponding risk curve and risk point of this model are shown in Figure 4. The point is below confidence curve but part of risk curve is above the confidence curve; thus the numerical experiment demonstrates that the optimal solution of this model is not the optimal solution of model (8) in this paper. 
TABLE 3: Optimal portfolios in three different situations (\%).

\begin{tabular}{lccccccccccc}
\hline Security $i$ & 1 & 2 & 3 & 4 & 5 & 6 & 7 & 8 & 9 \\
\hline Proportion 1 & 0.00 & 0.00 & 0.00 & 12.47 & 0.00 & 0.00 & 40.52 & 0.00 & 47.02 & 0.00 \\
Proportion 2 & 0.00 & 0.00 & 0.00 & 21.98 & 0.00 & 0.00 & 78.02 & 0.00 & 0.00 & 0.00 \\
Proportion 3 & 0.00 & 0.00 & 0.00 & 20.89 & 0.00 & 0.00 & 32.59 & 0.00 & 46.51 & 0.00 \\
\hline
\end{tabular}

Remark: Proportion 1 denotes the allocation proportion of the model with background risk and liquidity; Proportion 2 denotes the allocation proportion of the model with background risk but without liquidity; Proportion 3 denotes the allocation proportion of the model with liquidity but without background risk.

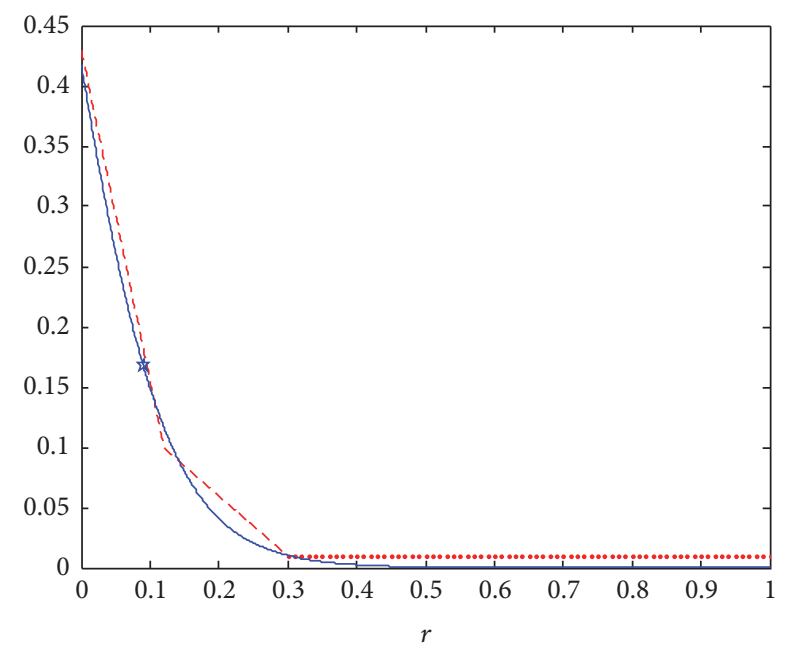

FIGURE 4: Risk point and risk curve of model (18).

\section{Conclusions}

In real life, background risk and asset liquidity may affect their investments in financial securities. In the complex financial and social environment, there are situations where background assets return, the security returns, and asset liquidity have to be evaluated by experienced experts due to the unexpected things and the lack of historical data.

This paper has discussed that risk level can be measured by risk curve and proposed uncertain portfolio selection models with background risk, asset liquidity, and transaction costs. The crisp equivalents of the model are provided. In addition, for any given $r$, many properties have been discussed to illustrate the influence of the background risk and asset liquidity on portfolio selection. Different from Huang's model, our model can consider all the possible risk that investors could tolerate.

Finally, both the numerical experiment results and the analysis show that the proposed models are effective and background risk and asset liquidity have a great effect on the optimal strategies.

\section{Appendix}

\section{Uncertainty Theory}

In this section, uncertain measure, uncertain variable, and uncertainty distribution will be introduced for easy understanding of the paper. In 2007, Liu [30] proposed uncertainty theory and got a wide range of applications. Firstly, we introduce the definition of uncertain measure.

Definition $A .1$ (see [30]). Let $L$ be $\sigma$-algebra over a nonempty set $\Omega$. A set function $M: L \rightarrow[0,1]$ is called an uncertain measure if it satisfies the following four axioms:

(1) (Normality) $M\{\Omega\}=1$.

(2) (Self-Duality) $M\{\Gamma\}+M\left\{\Gamma^{c}\right\}=1$.

(3) (Countable Subadditivity) For every countable sequence of events $\left\{\Gamma_{i}\right\}$, one has

$$
M\left\{\bigcup_{i=1}^{\infty} \Gamma_{i}\right\} \leq \sum_{i=1}^{\infty} M\left\{\Gamma_{i}\right\} .
$$

The triplet $(\Omega, L, M)$ is called an uncertainty space.

(4) (Product Measure [39]) For uncertainty spaces $\left(\Omega_{i}, L_{i}, M_{i}\right), i=1,2, \ldots$, the product uncertain measure is

$$
M\left\{\prod_{i=1}^{\infty} \Gamma_{i}\right\}=\bigwedge_{i=1}^{\infty} M_{i}\left\{\Gamma_{i}\right\}
$$

where $\Gamma_{i}$ are arbitrary chosen events from $L_{i}$ for $i=$ $1,2, \ldots$, respectively.

It is easy to prove that any uncertain measure $M$ is increasing. That is,

$$
M\left\{\Gamma_{1}\right\} \leq M\left\{\Gamma_{2}\right\}
$$

for any events $\Gamma_{1} \subset \Gamma_{2}$.

Definition A.2 (see [30]). An uncertain variable is a function $\xi:(\Omega, L, M) \rightarrow R$; that is, for any Borel set $B$ of real numbers, the set

$$
\{\xi \in B\}=\{\chi \in \Omega \mid \xi(\chi) \in B\}
$$

is an event.

Definition A.3 (see [30]). For any $x \in R$, the uncertainty distribution of an uncertain variable $\xi$ is defined as $\Phi(x)=$ $M\{\xi \leq x\}$. It is said to be regular if it is a continuous and strictly increasing function with respect to $x$ at which $0<$ $\Phi(x)<1$, and

$$
\begin{aligned}
& \lim _{x \rightarrow-\infty} \Phi(x)=0, \\
& \lim _{x \rightarrow+\infty} \Phi(x)=1 .
\end{aligned}
$$


The inverse function $\Phi^{-1}(\alpha)$ is called the inverse uncertainty distribution of $\xi$ if it exists and is unique for each $\alpha \in(0,1)$. Inverse uncertainty distribution plays a crucial role in the operations of independent uncertain variables.

Next we introduce some commonly used uncertainty distributions.

Definition A.4 (see [30]). An uncertain variable is called zigzag if it has a zigzag uncertainty distribution:

$$
\Phi(x)= \begin{cases}0, & \text { if } x \leq a, \\ \frac{(x-a)}{2(b-a)}, & \text { if } a \leq x \leq b, \\ \frac{(x+c-2 b)}{2(c-b)}, & \text { if } b \leq x \leq c, \\ 1, & \text { if } x \geq c,\end{cases}
$$

denoted by $Z(a, b, c)$, where $a, b, c$ are real numbers with $a<$ $b<c$.

Definition A.5 (see [30]). An uncertain variable is called normal if it has a normal uncertainty distribution:

$$
\Phi(x)=\left(1+\exp \left(\frac{\pi(e-x)}{\sqrt{3} \sigma}\right)\right)^{-1}, \quad x \in R
$$

denoted by $N(e, \sigma)$, where $e$ and $\sigma$ are real numbers with $\sigma>$ 0 .

Definition A.6 (see [40]). An uncertain variable $\xi$ is called empirical if it has an empirical uncertainty distribution:

$$
\begin{aligned}
& \Phi(x) \\
& = \begin{cases}0, & \text { if } x<x_{i}, \\
\alpha_{i}+\frac{\left(\alpha_{i+1}-\alpha_{i}\right)\left(x-x_{i}\right)}{x_{i+1}-x_{i}}, & \text { if } x_{i}<x<x_{i+1}, \quad 1 \leq i<n, \\
1, & \text { if } x>x_{n},\end{cases}
\end{aligned}
$$

where $x_{1}<x_{2}<\cdots<x_{n}$ and $0 \leq \alpha_{1} \leq \alpha_{2} \leq \cdots \leq \alpha_{n} \leq 1$.

Theorem A.7 (see [30]). Let $\xi_{1}, \xi_{2}, \ldots, \xi_{n}, \xi_{n+1}, \ldots, \xi_{n+m}$ be independent uncertain variables with continuous and strictly increasing uncertainty distribution functions $\Psi_{1}$, $\Psi_{2}, \ldots, \Psi_{n}, \Psi_{n+1}, \ldots, \Psi_{n+m}$, respectively. Let $f\left(x_{1}, x_{2}, \ldots\right.$, $\left.x_{n}, x_{n+1}, \ldots, x_{n+m}\right)$ be strictly increasing with respect to $x_{1}, x_{2}, \ldots, x_{n}$ and strictly decreasing with respect to $x_{n+1}$, $x_{n+2}, \ldots, x_{n+m}$. Then

$$
\xi=f\left(\xi_{1}, \xi_{2}, \ldots, \xi_{n}, \xi_{n+1}, \ldots, \xi_{n+m}\right)
$$

is an uncertain variable whose inverse uncertainty distribution function is

$$
\begin{gathered}
\Phi^{-1}(\alpha)=f\left(\Psi_{1}^{-1}(\alpha), \Psi_{2}^{-1}(\alpha), \ldots, \Psi_{n}^{-1}(\alpha),\right. \\
\left.\Psi_{n+1}^{-1}(1-\alpha), \ldots, \Psi_{n+m}^{-1}(1-\alpha)\right), \quad 0<\alpha<1 .
\end{gathered}
$$

Definition A.8 (see [30]). The expected value of an uncertain variable $\xi$ is defined by

$$
E[\xi]=\int_{0}^{+\infty} M\{\xi \geq x\} d x-\int_{-\infty}^{0} M\{\xi \leq x\} d x
$$

provided that at least one of the two integrals exists.

Theorem A.9 (see [30]). Let $\xi_{1}$ and $\xi_{2}$ be independent uncertain variables with finite expected values. Then for any real numbers $a_{1}$ and $a_{2}$, one has

$$
E\left[a_{1} \xi_{1}+a_{2} \xi_{2}\right]=a_{1} E\left[\xi_{1}\right]+a_{2} E\left[\xi_{2}\right] .
$$

\section{Competing Interests}

The authors declare that they have no competing interests.

\section{Acknowledgments}

This work was supported by National Natural Science Foundation of China [Grant nos. 71571007 and 71333014] and Aeronautical Science Foundation of China [Grant no. 2012ZG51079].

\section{References}

[1] H. Markowitz, "Portfolio selection," The Journal of Finance, vol. 7, no. 1, pp. 77-91, 1952.

[2] H. Markowitz, Portfolio Selection: Efficient Diversification of Investments, John Wiley \& Sons, New York, NY, USA, 1959.

[3] P. D. Kaplan and R. H. Alldredge, "Semivariance in risk-based index construction: quantidex global indexes," The Journal of Investing, vol. 6, no. 2, pp. 82-87, 1997.

[4] H. Konno and H. Yamazaki, "Mean-absolute deviation portfolio optimization model and its applications to Tokyo Stock Market," Management Science, vol. 37, no. 5, pp. 519-531, 1991.

[5] P. Jorion, "Measuring the risk in value at risk," Finance Analysts Journal, vol. 52, no. 6, pp. 47-56, 1996.

[6] R. Mansini, W. Ogryczak, and M. G. Speranza, "Conditional value at risk and related linear programming models for portfolio optimization," Annals of Operations Research, vol. 152, no. 1, pp. 227-256, 2007.

[7] A. A. Najafi and S. Mushakhian, "Multi-stage stochastic meansemivariance-CVaR portfolio optimization under transaction costs," Applied Mathematics and Computation, vol. 256, pp. 445458, 2015.

[8] X. Huang, "Portfolio selection with a new definition of risk," European Journal of Operational Research, vol. 186, no. 1, pp. 351357, 2008.

[9] X. Huang, "Mean-risk model for uncertain portfolio selection," Fuzzy Optimization and Decision Making, vol. 10, no. 1, pp. 7189, 2011.

[10] R. D. Arnott and W. H. Wagner, "The measurement and control of trading costs," Financial Analysts Journal, vol. 46, no. 6, pp. 73-80, 1990.

[11] M. A. Parra, A. B. Terol, and M. V. R. Urıa, "A fuzzy goal programming approach to portfolio selection," European Journal of Operational Research, vol. 133, no. 2, pp. 287-297, 2001. 
[12] Y. Fang, K. K. Lai, and S.-Y. Wang, "Portfolio rebalancing model with transaction costs based on fuzzy decision theory," European Journal of Operational Research, vol. 175, no. 2, pp. 879-893, 2006.

[13] J. Detemple and S. Sundaresan, "Nontraded asset valuation with portfolio constraints: a binomial approach," The Review of Financial Studies, vol. 12, no. 4, pp. 835-872, 1999.

[14] C. Gollier and J. W. Pratt, "Risk vulnerability and the tempering effect of background risk," Econometrica, vol. 64, no. 5, pp. 1109$1123,1996$.

[15] A. M. Baptista, "Optimal delegated portfolio management with background risk," Journal of Banking \& Finance, vol. 32, no. 6, pp. 977-985, 2008.

[16] A. M. Baptista, "Portfolio selection with mental accounts and background risk," Journal of Banking \& Finance, vol. 36, no. 4, pp. 968-980, 2012.

[17] J. Heaton and D. Lucas, "Portfolio choice and asset prices: the importance of entrepreneurial risk," The Journal of Finance, vol. 55, no. 3, pp. 1163-1198, 2000.

[18] H. S. Rosen and S. Wu, "Portfolio choice and health status," Journal of Financial Economics, vol. 72, no. 3, pp. 457-484, 2004.

[19] C. Hara, J. Huang, and C. Kuzmics, "Effects of background risks on cautiousness with an application to a portfolio choice problem," Journal of Economic Theory, vol. 146, no. 1, pp. 346358, 2011.

[20] B. Liu, "Why is there a need for uncertainty theory?" Journal of Uncertain Systems, vol. 6, no. 1, pp. 3-10, 2012.

[21] Q. Zhang, X. Huang, and C. Zhang, "A mean-risk index model for uncertain capital budgeting," Journal of the Operational Research Society, vol. 67, no. 4, pp. 1-10, 2014.

[22] L. A. Zadeh, "Fuzzy sets," Information and Computation, vol. 8, pp. 338-353, 1965.

[23] C. Carlsson and R. Fullér, "On possibilistic mean value and variance of fuzzy numbers," Fuzzy Sets and Systems, vol. 122, no. 2, pp. 315-326, 2001.

[24] L.-H. Chen and L. Huang, "Portfolio optimization of equity mutual funds with fuzzy return rates and risks," Expert Systems with Applications, vol. 36, no. 2, pp. 3720-3727, 2009.

[25] X. Huang, "Portfolio selection with fuzzy returns," Journal of Intelligent and Fuzzy Systems, vol. 18, no. 4, pp. 383-390, 2007.

[26] X. Huang, "Mean-semivariance models for fuzzy portfolio selection," Journal of Computational and Applied Mathematics, vol. 217, no. 1, pp. 1-8, 2008.

[27] Z. Qin, X. Li, and X. Ji, "Portfolio selection based on fuzzy crossentropy," Journal of Computational and Applied Mathematics, vol. 228, no. 1, pp. 139-149, 2009.

[28] X. Li, Z. Qin, and S. Kar, "Mean-variance-skewness model for portfolio selection with fuzzy returns," European Journal of Operational Research, vol. 202, no. 1, pp. 239-247, 2010.

[29] R. Bhattacharyya, S. Kar, and D. D. Majumder, "Fuzzy meanvariance-skewness portfolio selection models by interval analysis," Computers and Mathematics with Applications, vol. 61, no. 1, pp. 126-137, 2011.

[30] B. Liu, Uncertainty Theory, Springer, Berlin, Germany, 2007.

[31] X. Huang, Portfolio Analysis: From Probabilistic to Credibilistic and Uncertain Approcches, Springer, Berlin, Germany, 2010.

[32] X. Huang, "A risk index model for portfolio selection with returns subject to experts' estimations," Fuzzy Optimization and Decision Making, vol. 11, no. 4, pp. 451-463, 2012.
[33] X. Huang, "Mean-variance models for portfolio selection subject to experts' estimations," Expert Systems with Applications, vol. 39, no. 5, pp. 5887-5893, 2012.

[34] X. Huang, T. Zhao, and S. Kudratova, "Uncertain meanvariance and mean-semivariance models for optimal project selection and scheduling," Knowledge-Based Systems, vol. 93, pp. 1-11, 2016.

[35] X. Huang and T. Zhao, "Mean-chance model for portfolio selection based on uncertain measure," Insurance: Mathematics and Economics, vol. 59, pp. 243-250, 2014.

[36] Z. Qin, "Mean-variance model for portfolio optimization problem in the simultaneous presence of random and uncertain returns," European Journal of Operational Research, vol. 245, no. 2, pp. 480-488, 2015.

[37] X. Huang and H. Di, "Uncertain portfolio selection with background risk," Applied Mathematics and Computation, vol. 276, pp. 284-296, 2016.

[38] C. Jiang, Y. Ma, and Y. An, "An analysis of portfolio selection with background risk," Journal of Banking \& Finance, vol. 34, no. 12, pp. 3055-3060, 2010.

[39] B. Liu, "Some research problems in uncertainty theory," Journal of Uncertain Systems, vol. 1, no. 3, pp. 3-10, 2009.

[40] B. Liu, Uncertainty Theory, Uncertainty Theory Laboratory, 5th edition, 2016. 


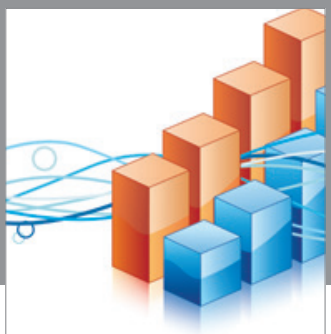

Advances in

Operations Research

vatem alat4

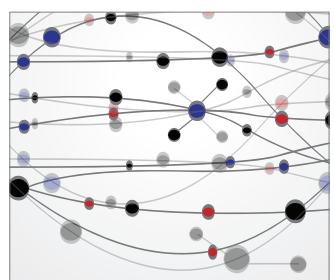

\section{The Scientific} World Journal
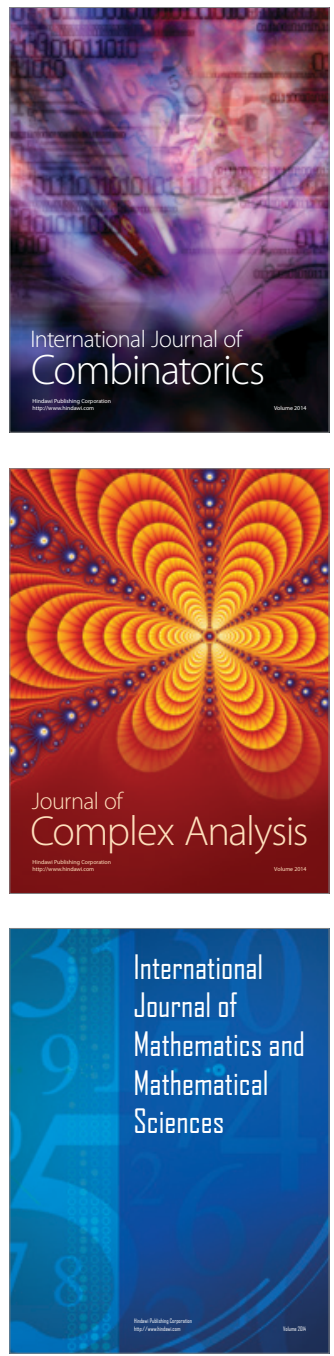
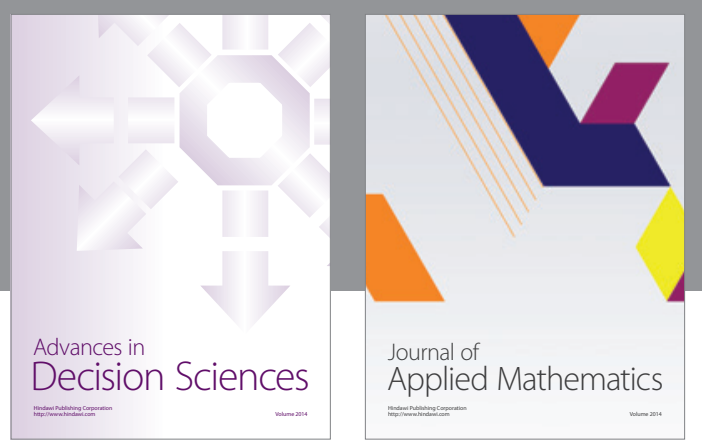

Algebra

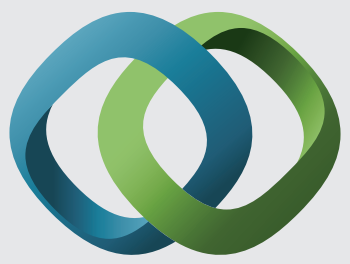

\section{Hindawi}

Submit your manuscripts at

https://www.hindawi.com
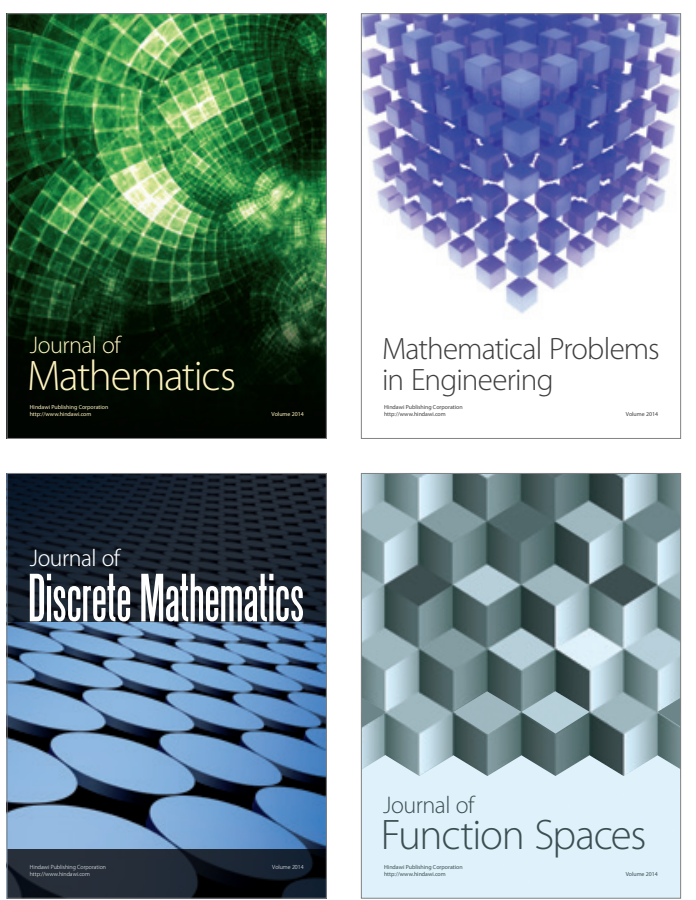

Mathematical Problems in Engineering
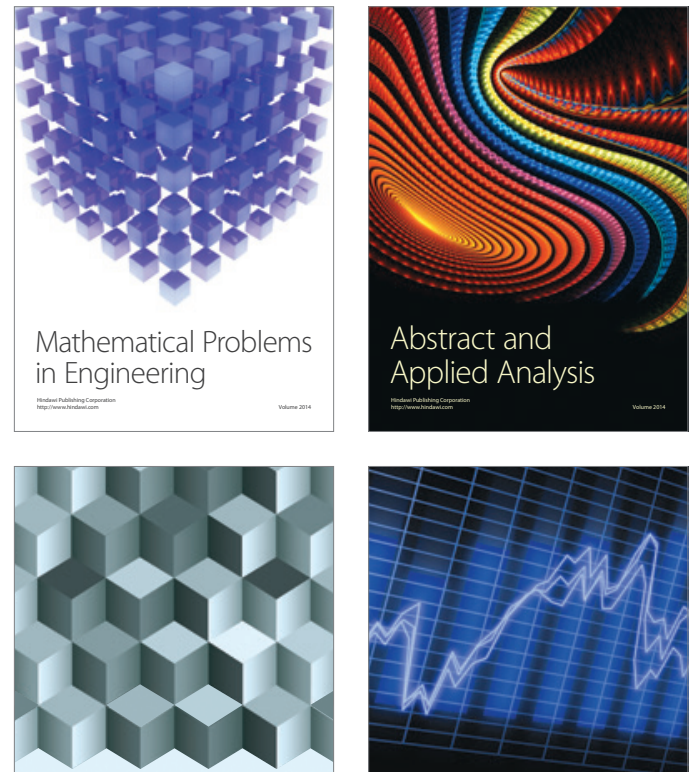

Journal of

Function Spaces

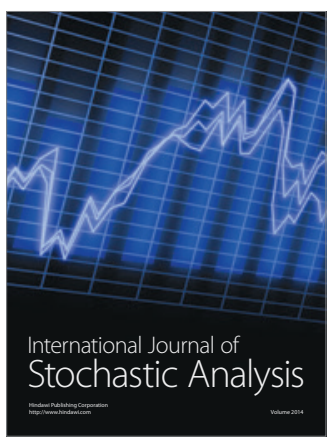

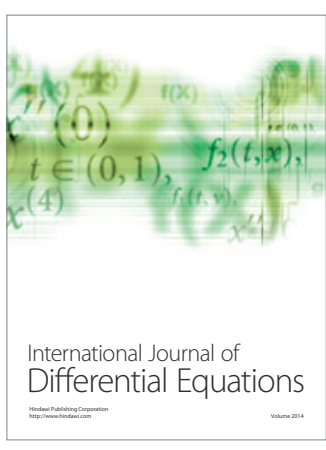
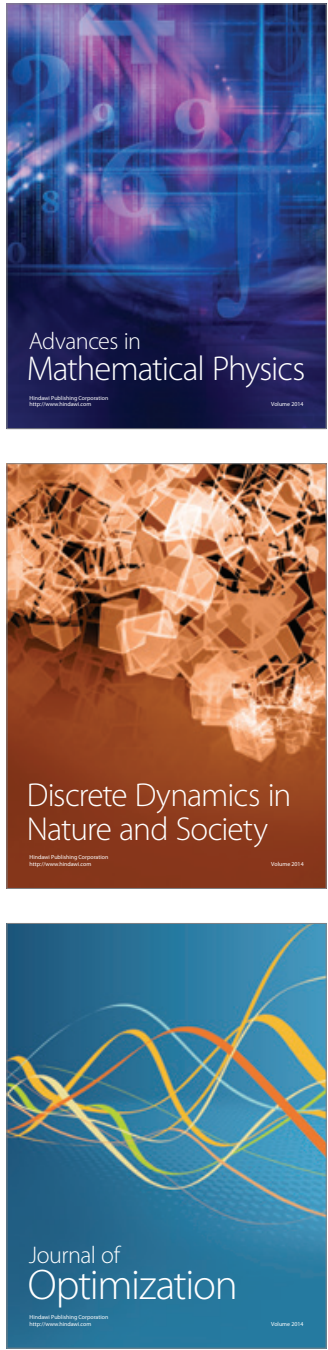